\title{
Efficacy and Safety of Transcutaneous Electrical Acupoint Stimulation (TEAS) for Postoperative Pain in Laparoscopy: A Systematic Review and Meta-Analysis of Randomized Controlled Trials
}

\author{
Dan Meng, ${ }^{1}$ Yifei Mao, ${ }^{1}$ Quan-mei Song, ${ }^{2}$ Chun-chun Yan, ${ }^{2}$ Qin-yu Zhao, Mengqi Yang $\mathbb{D},{ }^{3}$ \\ Guangxin Xiang, ${ }^{1}$ and Yongmei Song $\mathbb{D}^{1}$ \\ ${ }^{1}$ Institute of Literature and Culture of Traditional Chinese Medicine, Shandong University of Traditional Chinese Medicine, \\ Jinan, Shandong 250355, China \\ ${ }^{2}$ Institute of Acupuncture, Moxibustion, and Massage, Shandong University of Traditional Chinese Medicine, Jinan, \\ Shandong 250355, China \\ ${ }^{3}$ Institute of Traditional Chinese Medicine, Shandong University of Traditional Chinese Medicine, Jinan, \\ Shandong 250355, China \\ Correspondence should be addressed to Yongmei Song; songym0200@163.com
}

Received 24 March 2021; Accepted 2 December 2021; Published 15 January 2022

Academic Editor: Talha Bin Emran

Copyright (c) 2022 Dan Meng et al. This is an open access article distributed under the Creative Commons Attribution License, which permits unrestricted use, distribution, and reproduction in any medium, provided the original work is properly cited.

\begin{abstract}
Objectives. This meta-analysis aimed to assess the efficacy and safety of transcutaneous acupoint electrical stimulation (TEAS) for postoperative pain in laparoscopy. The review has been registered on the "INPLASY" website and the registration number is INPLASY202150101. Methods. Relevant randomized controlled trials are selected from seven electronic databases (PubMed, the Cochrane Library, Embase, China National Knowledge Infrastructure, Chongqing VIP Information, WanFang Data, and Chinese Biomedical Database) from their inception up to November 30, 2020. Twenty-eight studies were included in this meta-analysis, and the statistical analyses and the exploration of heterogeneity sources were conducted by Stata 15.0 software. Besides, the bias assessment of the included studies was evaluated using the Cochrane risk of bias tool. Results. In total, 28 RCTs covering 2787 participants were included. The meta-analysis suggested that TEAS can effectively relieve pain in the short term after laparoscopy, reduce the postoperative consumption of rescue analgesics, improve the quality of life of patients, and shorten the length of hospitalization. And no serious adverse events are related to TEAS. Therefore, TEAS is relatively safe and efficacy for clinical application. The most used acupoints were Hegu (LI14), Neiguan (PC6), and Zusanli (ST36). Conclusions. TEAS can be recommended as a complementary and alternative therapy for the treatment of postoperative pain after laparoscopy. However, the included RCTs had some methodological limitations. Therefore, larger-size, more rigorous, and higher-quality RCTs are needed in the future to further explore the efficacy and safety of TEAS for postoperative pain after laparoscopy.
\end{abstract}

\section{Introduction}

With the promotion of the concept of minimally invasive surgery, the application of laparoscopy has gradually increased because of its advantages of small trauma to surrounding tissues, less intraoperative bleeding, rapid postoperative recovery, and a wide application range. However, various types of pain such as incision pain, visceral pain, and nonincision pain often occur after laparoscopy, among which shoulder and back pain, diaphragm pain, intercostal muscle pain, and other nonoperative incision pain are more common [1]. This is a serious complication after laparoscopy, which is called "postlaparoscopic pain syndrome" [2]. It may be related to the following factors: the inflammatory injury or traction of the phrenic nerve caused by the residual $\mathrm{CO}_{2}$ in the abdomen after the surgery, the 
proliferation of peritoneal inflammation induced by the acid environment in the abdomen, the humidity and temperature of the pneumoperitoneum, and the position and types of the surgery [3-7].

To some extent, postoperative pain may produce a negative effect on patients' physical activity, recovery time, quality of life, medical expenses, etc. Therefore, optimizing the management of postoperative acute pain is crucial for accelerating postoperative rehabilitation. Though a variety of analgesic methods, such as nonsteroidal anti-inflammatory drugs (NSAIDs) or COX-2 analgesic drugs, as well as epidural analgesia, patient-controlled intravenous analgesia (PCIA), and nerve block, have been widely used in the clinic, they can also produce more side effects. For example, opioids can lead to postoperative nausea and vomiting, skin pruritus, respiratory inhibition, and other adverse reactions [8]. Therefore, to reduce the side effects, many researchers began to turn to complementary and alternative therapies. One study [9] found that acupuncture and related techniques can significantly reduce postoperative pain score and opioid consumption and can be used as an effective auxiliary means of postoperative pain management. One study [10] reported that TEAS can significantly relieve postoperative pain and reduce opioid consumption on the first postoperative day. Another study [11] limited the mode of acupuncture to the way needling with penetration of the skin and only investigated the efficacy of such way on postoperative pain after laparoscopy and did not analyze the noninvasive acupoint stimulation mode.

Transcutaneous acupoint electrical stimulation (TEAS) is a noninvasive treatment formed by the gradual improvement of traditional acupuncture and moxibustion. During the operation, the specific low-frequency pulse current is input into the human body through the electrodes placed on the acupoints' surface and stimulates the acupoint to achieve a certain therapeutic effect, which combines the advantages of acupoint therapy and transcutaneous electric nerve stimulation (TENS). It can reduce the incidence of infection and bleeding, can be controlled or reused by patients and be operated by the operator after simple training, and has good treatment compliance. Many clinical randomized controlled trials (RCTs) have reported transdermal electrical stimulation is widely used in surgeries and plays a positive role in the treatment of postoperative pain [12-14]. In addition, pharmacological studies have shown that some medicinal plants are safe natural analgesics and have potential therapeutic advantages. For example, Molineria capitulata and Spirulina platensis have anti-inflammatory, antioxidant, and analgesic effects $[15,16]$. Bromelain is a natural drug to alleviate the symptoms of arthritis (including joint pain and stiffness) [17]. It has an active therapeutic effect on immune system diseases. Flavonoids can play an auxiliary role in the treatment of pain in the digestive system and nervous system [18]. These studies may bring new clinical ideas to the treatment of postoperative pain. However, this time we are only going to evaluate the efficacy and safety of TEAS in the treatment of postoperative pain after laparoscopy, so we conducted this systematic review and meta-analysis. To our knowledge, this is the first metaanalysis focusing on the outcomes of TEAS in the treatment of postoperative pain after laparoscopy.

\section{Materials and Methods}

This systematic review and meta-analysis was performed in line with the Preferred Reporting Items for Systematic Reviews and Meta-Analyses (PRISMA) schema and was registered at INPLASY under registration number INPLASY202150101, and the corresponding protocol has been published in Medicine [19].

2.1. Data Sources and Search Strategy. A comprehensive search was carried out in PubMed, the Cochrane Library (CENTRAL), Embase, and four Chinese databases (China National Knowledge Infrastructure, Chongqing VIP Information, WanFang Data, and Chinese Biomedical Database) from their inception up to November 30, 2020. Because of the language restriction of our researchers, only studies published in English and Chinese were included. The search strategy consisted of Medical Subject Heading (Mesh) terms and free-text terms with logical operators. Asterisks are truncation symbols that can help us search for all designs with asterisks. We performed an initial search of PubMed as follows (Table 1): \#1 "transcutaneous electrical acupoint stimulation" OR "transcutaneous acupoint electrical stimulation" OR "electr* stimulat*" OR "electr* acustimul*" OR "electroacupuncture*" OR "electro-acupuncture" OR "TEAS"; \#2 "Laparoscopy[Mesh]" OR "laparoscop*” OR "coelioscop*" OR “celioscop*" OR "peritoneoscop*"; \#3 "Pain, Postoperative[Mesh]" OR "postoperative pain" OR "postoperative analgesi*" OR "pain management" OR "ache*” OR "suffering*” OR "discomfort." We also further searched the grey literature and the retrieved references to avoid omission. For the literature to be difficult to obtain the full text, we checked and identified the ongoing or unpublished studies through the World Health Organization International Clinical Trials Registry Platform (WHO ICTRP), Clinical Trials.gov, Chinese Clinical Trial Registry (Chi CTR), and the reference list of eligible RCTs. Two reviewers (Meng and Mao) independently screened the titles and abstracts for eligibility and examined the full text of the articles. Any discrepancies were resolved by consensus or after consulting a third party (Song).

\subsection{Study Selection}

\subsubsection{Inclusion Criteria of Studies}

(1) Participants: they were adults over 18 years old and underwent any kind of laparoscopy under general anesthesia. There are no restrictions on gender, race, occupation, location, malignancy of the disease, and many other aspects. When these patients underwent surgery other than laparoscopy, we only analyzed the pain indicators related to laparoscopy. 
TABle 1: Search strategy in PubMed up till November 30, 2020 (similar search run in other databases).

\begin{tabular}{|c|c|}
\hline$\# 1$ & $\begin{array}{l}\text { "transcutaneous electrical acupoint stimulation" OR "transcutaneous acupoint electrical stimulation" OR "electr* stimulat*" OR } \\
\text { "electr* acustimul*" OR "electroacupuncture*" OR "electro-acupuncture" OR "TEAS" }\end{array}$ \\
\hline$\# 2$ & “Laparoscopy[Mesh]” OR "laparoscop*” OR “coelioscop*” OR “celioscop*” OR "peritoneoscop*” \\
\hline \#3 & "Pain, Postoperative[Mesh]" OR "postoperative pain" OR "postoperative analgesi*" OR "pain management" OR "ache*" OR \\
\hline$\# 4$ & \#1 AND \#2 AND \#3 \\
\hline
\end{tabular}

(2) Type of study: randomized controlled trials (RCTs) in English or Chinese with no limitation of the blinding method.

(3) Type of interventions: the intervention groups were treated with TEAS or TEAS combined with other therapy, such as patient-controlled intravenous analgesia (PCIA) or other anesthesia methods. The intervention time, frequency, waveform, acupoints, and course of treatment of TEAS are not limited. The control group were treated with sham-TEAS or blank control or combined with other treatment methods which are the same as the intervention group or combination of the above several methods.

(4) Study outcomes: primary measures: (a) pain intensity: relevant overall postoperative pain using any scale, such as Visual Analogue Scale (VAS); (b) consumption of postoperative analgesics. Secondary measures: (a) quality of life after the surgery: assessed using validated scales, such as the pain score in QoL40; (b) duration of hospitalization; (c) adverse events such as postoperative nausea, vomiting, pruritus, etc. Safety evaluation was assessed using the adverse event reported in the studies

\subsubsection{Exclusion Criteria of Studies}

(1) Non-RCTs, crossover trials, quasi-randomized trials, protocols, reviews, case reports, animal experimental research studies, ongoing trials, literature without full text, duplicate publications, and irrelative interventions and outcomes were excluded.

(2) We excluded trials in which the controls underwent different frequency, waveform, intervention time, and other forms of TEAS compared with the intervention group.

2.3. Data Extraction and Quality Assessment. Data extraction was independently conducted by two investigators (Meng and Mao) according to the criteria above. Disagreements were resolved through discussion and consensus was reached with a third investigator (Yan). The two authors made a final judgment by reading the full text of the remaining articles. All available information related to our research was extracted from the included studies. The following data was extracted from the studies using a predesigned form: first author name, publication year, types of surgery, sample size, intervention, intervention measures and time, acupoints, waveform, electrical stimulation frequency, outcome measures, and adverse events. If the information was incomplete, we tried to acquire it by contacting the correspondent authors via e-mail or telephone.

The Cochrane risk of bias tool [20] was used to assess the methodological qualities of the included trials by the two reviewers (Meng and Mao). The contents included random sequence generation, allocation concealment, blinding of participants and personnel, blinding of outcome assessment, selective reporting, incomplete outcome data, and other risks of bias. The risk of bias was classified as "low-risk," "high-risk," or "unclear-risk." Disagreements in this interpretation were resolved via consensus or after discussion with a third party (Song).

2.4. Data Analysis. Statistical analysis was performed with the Stata 15.0 software. $P<0.05$ was considered statistically significant. For continuous variables, so the mean difference (MD) or standard mean difference (SWD) with 95\% confidence interval (CI) was used for analysis. For dichotomous data, such as the rates of responders and adverse events, the relative risk (RR) with $95 \%$ confidence intervals (CIs) was utilized for analysis. In the multiarm RCTs, we only extracted the data related to our study. For studies that satisfied the predefined inclusion criteria with multiple intervention groups, which used different intervention duration or different acupoints, meta-analysis will be conducted carefully when merged the data into a unified intervention group data. The magnitude of heterogeneity was measured using heterogeneity index $I^{2}$ statistic: when $I^{2}<50 \%$, a fixedeffects model will be used for pooled data; when $I^{2} \geq 50 \%$, a random-effects model was used. For each merged analysis, a heterogeneity test was performed using the chi-squared statistic. If $I^{2} \geq 50 \%$, the synthesized studies were considered an indicator of a substantial level of heterogeneity. Subgroup or sensitivity analysis was performed to identify the cause. Subgroup analyses identified the possible factors that contributed to the heterogeneity, such as different intervention duration, interventions, types of surgery, and waveform. Funnel plots were introduced to detect the existence of potential publication bias within RCTs $(n \geq 10)$. If metaanalysis is not feasible, narrative descriptions are provided. If heterogeneity of significance found in the included studies cannot be explained by subgroup analysis, we will not conduct a meta-analysis.

\section{Results}

3.1. Search Results. A total of 238 articles were identified during the initial study selection, 82 duplicates were removed, and 121 articles were excluded after reviewing 
abstracts and titles. Seven articles were excluded after reading the full text. For articles in which the data were repeatedly published, we choose the one with the higher quality and more related outcomes. Finally, 28 RCTs [21-48] met the eligibility criteria and were included in the systematic review. Standard general anesthesia was used in all trials. The PRISMA flow chart showed the study selection process in Figure 1.

3.2. Characteristics of Studies Included in the Review. In the included 28 trials [21-48], 4 [22, 23, 34, 47] were published in English, and the others were published in Chinese. There were 1627 participants in the intervention group and 1160 participants in the control group. All of the included studies were conducted in China and showed no significant difference within the baseline. The surgery types were different among these trials, much of them underwent gynecological surgery $[22,24,26,28,33-35,37,40,41,43,44,46]$ and gastrointestinal surgery [26, 29-31, 42, 45, 47, 48]. In the treatment groups, 18 studies [21-38] adopted TEAS alone, 5 studies [39-43] adopted TEAS with PCIA/PCEA, and 5 studies [44-48] adopted TEAS combined with other treatments. In the control groups, 11 studies [21-31] adopted sham-TEAS, 7 studies [33-38, 44] adopted blank control, and 10 studies [32, 39-43, 45-48] adopted the same measures as intervention groups besides TEAS. There is a total of 14 acupoints, in which the most common acupoints are Zusanli (ST36), Nieguan (PC6), and Hegu (LI14). The intervention time was different and the duration from the time before anesthesia induction to the end of the operation was most commonly used. As we can see, the dilatational waveform and continuous waveform are commonly used. For stimulation frequency, $2 / 100 \mathrm{~Hz}$ and $2 / 10 \mathrm{~Hz}$ were popular among the trials. The main characteristics and the specific interventions of each identified study are presented in Table 2.

3.3. Quality Assessment. Figure 2 summarizes the risk of bias in the 28 eligible RCTs, and the grade of the risk was recorded as "high-risk," "low-risk," and "unclear-risk." In terms of random sequence generation, 23 studies $[21-35,39,41-44,46-48]$ were considered as "low-risk" for they reported explicit random sequence methods, such as random number table method or computer-generated random number while others [36-38, 40,45] were considered as "unclear-risk" for they only mentioned "random" or "randomization" and did not report the specific random sequence method. In terms of the methods of allocation concealment, 7 studies [22, 24, 28, 32, 43, 47, 48] were considered as "low-risk" for they reported the method of assigning concealment, while others did not report it. 10 studies [22-24, 27-29, 32, 34, 43, 47] were considered as "low-risk" which performed a blinding method on subjects and operators. 7 studies [22, 24, 28, 32, 40, 43, 47] were considered as "low-risk" which performed a blinding method on results evaluators. 27 eligible RCTs reported the planned outcomes, so they were associated with a "low risk" of bias for blinding of selective reporting while one study
[47] was considered as "unclear risk" for it did not report the predesigned outcomes in the research protocol. One study [47] did not report missing data and was considered as "unclear risk," while other studies were considered as "low risk" for they reported the number and reasons of dropout members or no dropout members were in their studies. Additionally, the included 28 eligible RCTs did not report any details about potential sources of bias; therefore, they were considered as "unclear risk" in other biases.

\subsection{Primary Outcomes}

3.4.1. Visual Analogue Scale (VAS). Among the included studies, 8 studies were reported only in the form of graphs, without mean and standard deviation. However, our contact with the author is often unsuccessful. Therefore, the data are often unavailable and can only be excluded. One study [43] recorded the mean VAS score and the most painful VAS score within $24 \mathrm{~h}$ after surgery. One study [26] recorded the incidence of shoulder pain $48 \mathrm{~h}$ after surgery. A study [23] recorded the postoperative Verbal Rating Scale (VRS) at different times. One study [36] reported 2 cases of VAS $\geq 4$ in postoperative adverse reactions. Due to the insufficient number of studies reporting these data, no meta-analysis could be completed. Seventeen studies $[25,27,29-$ $31,34,35,37-42,44-46,48]$ recorded VAS scores at different times after the surgery. Therefore, to improve the validity of evidence, only studies ( $n \geq 5$ RCTs) reported VAS scores were analyzed to explore the analgesic effect of TEAS after laparoscopy.

Five studies reported a postoperative VAS score at $4 \mathrm{~h}$ (Figure 3). The fixed-effects model was used with good homogeneity $\left(P=0.248, I^{2}=26.1 \%\right)$. The combined data showed that the TEAS group was effective in reducing pain at this time compared with the control group $[\mathrm{WMD}=-0.54,95 \% \mathrm{CI}(-0.66,-0.43), P<0.001]$. Six studies reported postoperative VAS scores at $6 \mathrm{~h}$ (Figure 4). Due to obvious heterogeneity $\left(P<0.001, I^{2}=94.0 \%\right)$, a randomeffects model was used and showed no statistical difference between the two groups. The combined data extracted from 9 studies related to postoperative VAS score $12 \mathrm{~h}$ showed obvious heterogeneity $\left(P<0.001, I^{2}=73.8 \%\right)$, and a randomeffects model was used. Based on the types of surgery, gastrointestinal surgery showed good intergroup homogeneity $\left(P=0.127, I^{2}=47.4 \%\right)$. The subgroup analysis in favor of the TEAS group was effective in reducing pain at this time compared with the control group in the gastrointestinal surgery and gynecological surgery $[\mathrm{WMD}=-0.25,95 \% \mathrm{CI}$ $(-0.43,-0.06), P=0.009 ; \mathrm{WMD}=-0.59,95 \%$ CI $(-0.87$, -0.32 ), $P<0.001$ ] (Figure 5). Fifteen studies (Figure 6) provided data related to postoperative VAS score at $24 \mathrm{~h}$ and a random-effects model was used for a statistical analysis because of obvious heterogeneity $\left(P<0.001, I^{2}=88.2 \%\right)$. Based on the waveform, we conducted a subgroup analysis. The homogeneity between continuous waveform groups is $\operatorname{good}\left(P=0.434, I^{2}=0 \%\right)$. The results showed significant pain reduction in favor of the TEAS group versus the control group at this time when applied continuous wave and 


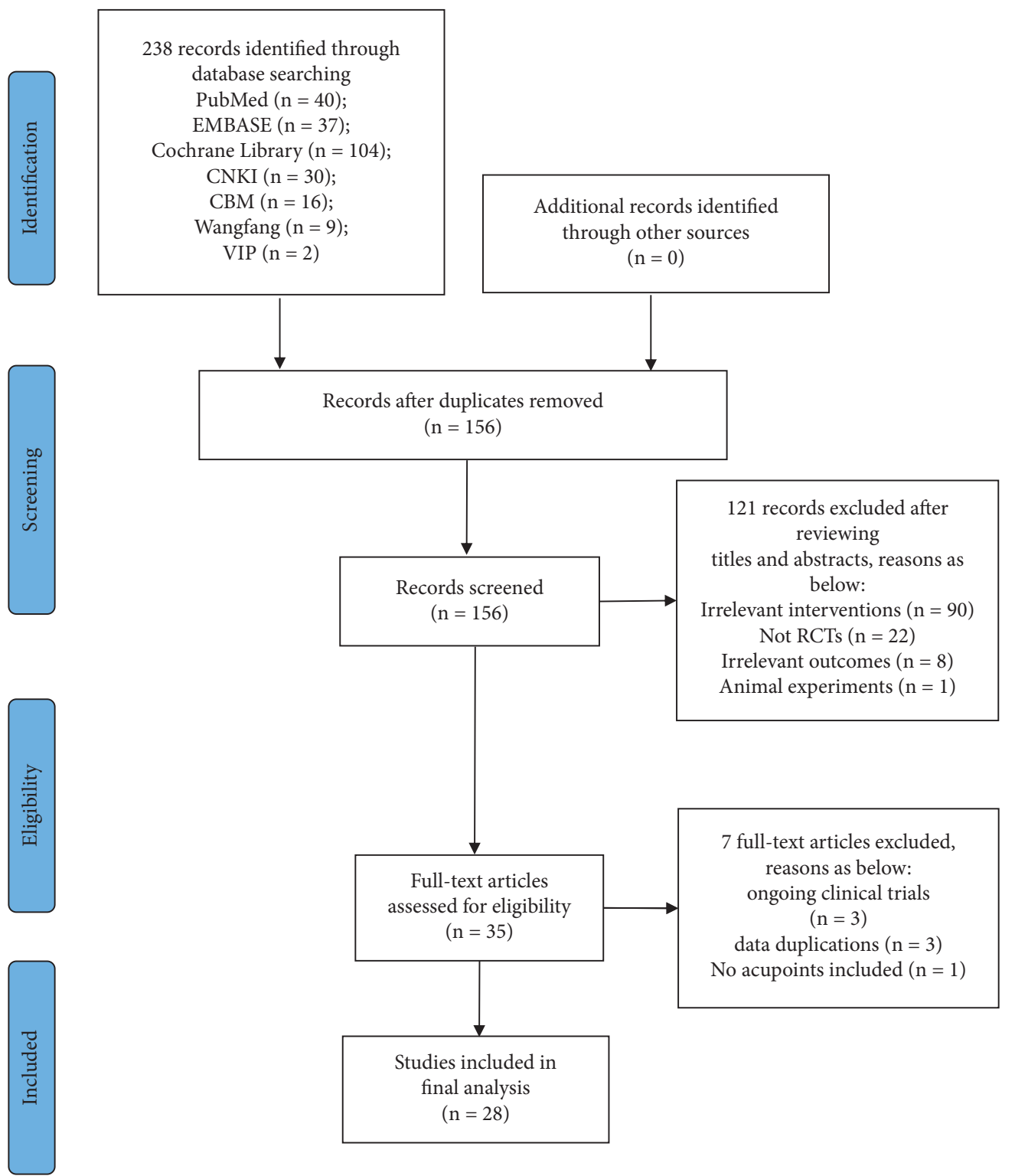

FIGURE 1: PRISMA flow diagram of the study process. PRISMA = preferred reporting items for systematic review and meta-analysis.

dilatational wave $[\mathrm{WMD}=-1.02,95 \% \mathrm{CI}(-1.27,-0.76)$, $P<0.001 ; \mathrm{WMD}=-0.78,95 \%$ CI $(-1.03,-0.53), P<0.001]$.

3.4.2. The Postoperative Consumption of Analgesics. In our study, the consumption of postoperative analgesics after laparoscopy was used as another important indicator to evaluate the analgesic effect of TEAS. Six studies $[21,29,30,38,41,42]$ reported it. After our analysis, the postoperative consumption of analgesics $12 \mathrm{~h}$ was suitable for meta-analysis. Three studies [29, 30, 41] reported this outcome. The fixed-effects model (Figure 7) was used according to no significant homogeneity $\left(P=0.943, I^{2}=0 \%\right)$. The combined data indicated that the postoperative consumption of analgesics was significantly lower in the TEAS group than that in the control group $[\mathrm{SMD}=-0.87,95 \% \mathrm{CI}$ $(-1.12,-0.63), P<0.001]$.

\subsection{Secondary Outcomes}

3.5.1. QoR-40 (Quality of Recovery-40) Score. Five studies $[21,22,34,43]$ reported the quality of recovery after surgery, in which four studies used the QoR-40 scoring scale, and one study [28] used the QoR-15 scoring scale. Four studies $[21,22,34,43]$ reported postoperative QoR-40 scores at different times, in which one study [28] did not record in the form of mean \pm standard deviation, so we excluded it. Based on the analysis of the data, the data of $24 \mathrm{~h}$ after surgery were considered suitable for meta-analysis.

Three studies [21, 22, 43] reported this outcome. A random-effects model (Figure 8) was selected due to the obvious heterogeneity $\left(P=0.018, I^{2}=75.2 \%\right)$. According to different types of surgery, the subgroup analysis was performed. It showed good homogeneity $\left(P=1.000, I^{2}=0 \%\right)$ and significant improvement $[\mathrm{WMD}=2.50,95 \% \mathrm{CI}(1.50$, 


\begin{tabular}{|c|c|c|c|c|c|c|c|c|c|c|c|c|}
\hline 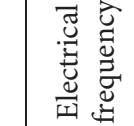 & 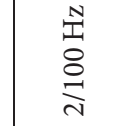 & 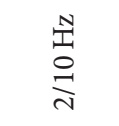 & $\begin{array}{l}\stackrel{N}{1} \\
\stackrel{0}{2} \\
\stackrel{\lambda}{N}\end{array}$ & 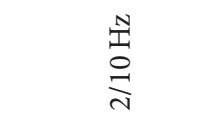 & $\underset{\substack{N \\
\stackrel{N}{N}}}{\stackrel{N}{N}}$ & $\stackrel{\substack{1 \\
8}}{\stackrel{2}{\lambda}}$ & 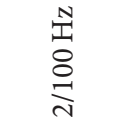 & $\stackrel{\text { N }}{\stackrel{N}{N}}$ & $\underset{\substack{N \\
8}}{\stackrel{N}{\lambda}}$ & 营 & $\stackrel{\substack{1 \\
8}}{\frac{1}{1 n}}$ & 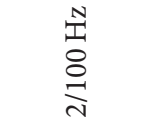 \\
\hline 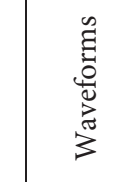 & 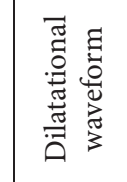 & 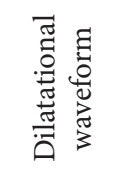 & 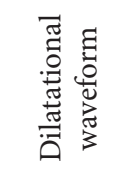 & 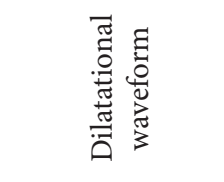 & 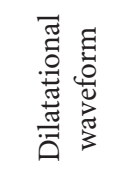 & 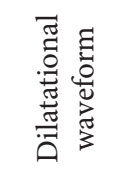 & 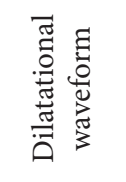 & 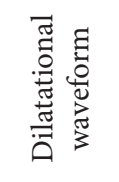 & 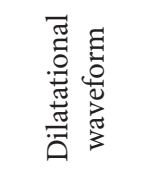 & 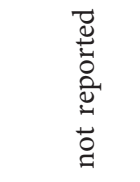 & 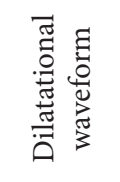 & 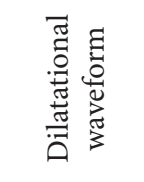 \\
\hline 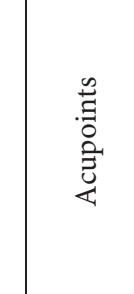 & 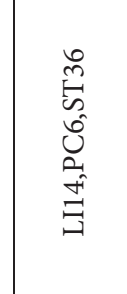 & 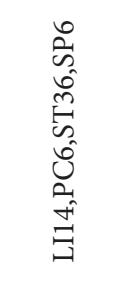 & ְ̊ & 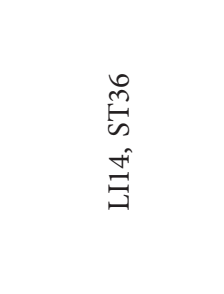 & $\begin{array}{l}0 \\
0 \\
0 \\
0 \\
0 \\
\omega \\
\omega\end{array}$ & $\begin{array}{l}0 \\
m \\
\omega_{n}^{2} \\
\bigcup_{0}^{2}\end{array}$ & $\begin{array}{l}\vec{J} \\
\vec{\exists} \\
\text { పे } \\
\text { के }\end{array}$ & 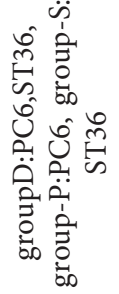 & $\begin{array}{l}0 \\
0 \\
0 \\
0 \\
\tilde{\omega} \\
\omega \\
\omega\end{array}$ & $\begin{array}{l}0 \\
0 \\
0 \\
0 \\
0 \\
0 \\
0 \\
0 \\
0 \\
0.1 \\
0 \\
0 \\
0 \\
0\end{array}$ & 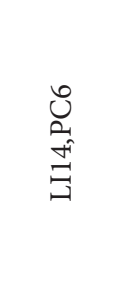 & 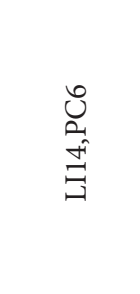 \\
\hline 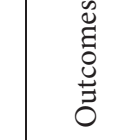 & $\stackrel{0}{\text { () }}$ & $\stackrel{\oplus}{\stackrel{(0)}{\odot}}$ & $\odot$ & $\Theta$ & $\odot$ & (๑) & $\odot$ & $\stackrel{\oplus}{\ominus}$ & $\stackrel{(0)}{\oplus}$ & (ㄱ) & $\odot$ & $\stackrel{\oplus}{\ominus}$ \\
\hline 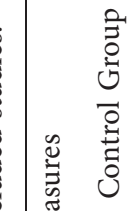 & 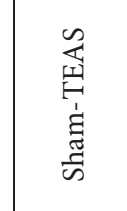 & 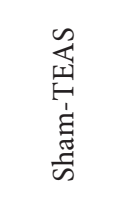 & 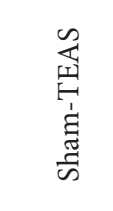 & 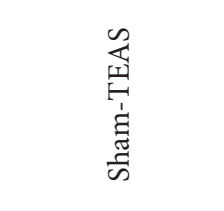 & 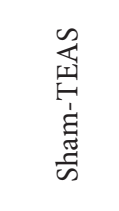 & 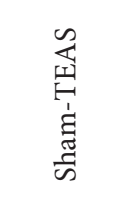 & 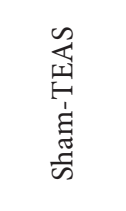 & 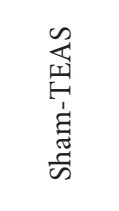 & 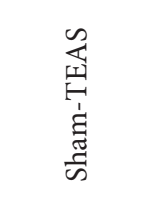 & 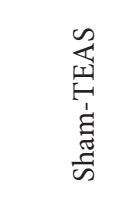 & 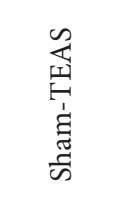 & 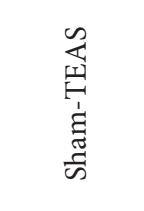 \\
\hline 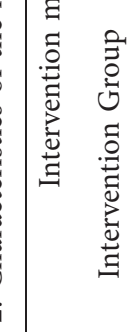 & $\begin{array}{l}\text { 足 } \\
\text { 至 }\end{array}$ & 武 & 空 & 离 & 死 & 离 & 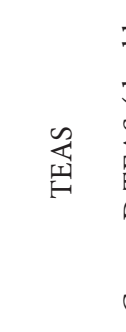 & 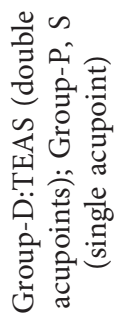 & 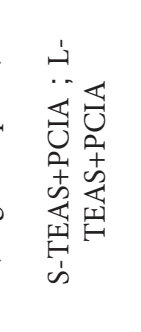 & 离 & 矛 & 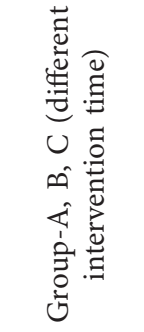 \\
\hline 尝营 & $\stackrel{\circ}{\circ}$ & $\frac{\stackrel{n}{m}}{\stackrel{m}{m}}$ & $\underset{\substack{\infty \\
+\infty}}{\infty}$ & $\begin{array}{l}\vec{m} \\
\vec{m}\end{array}$ & $\stackrel{\stackrel{\llcorner}{\Im}}{\underset{\sim}{\sim}}$ & 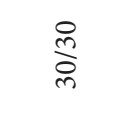 & 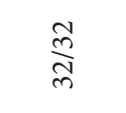 & 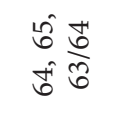 & $\begin{array}{l}\bar{q} \\
\hat{q} \\
\hat{q}\end{array}$ & $\frac{8}{\frac{8}{6}}$ & $\stackrel{\stackrel{\sim}{N}}{\stackrel{N}{N}}$ & $\begin{array}{l}\hat{\sigma} \& \\
\dot{\sigma} \infty\end{array}$ \\
\hline 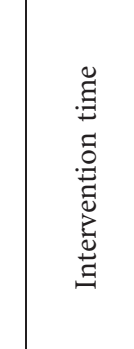 & 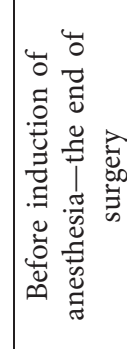 & 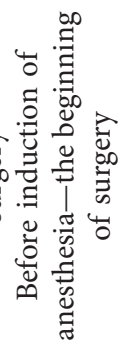 & 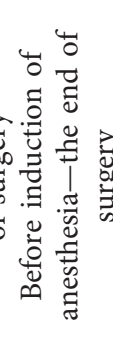 & 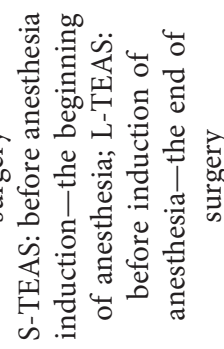 & 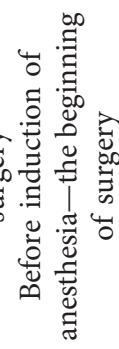 & 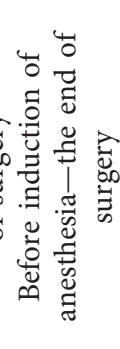 & 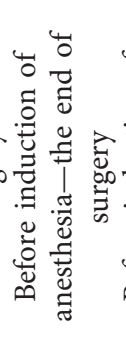 & 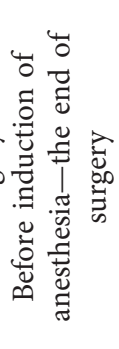 & 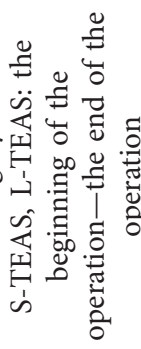 & 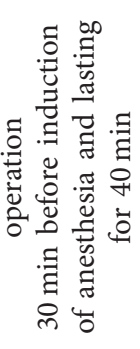 & 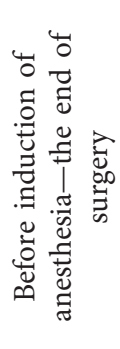 & 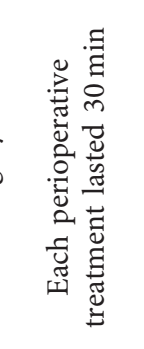 \\
\hline 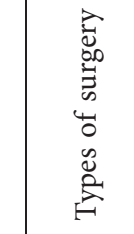 & 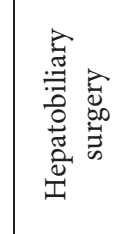 & 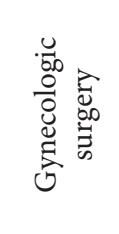 & 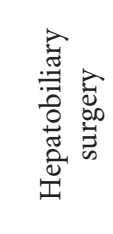 & 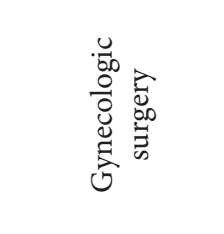 & 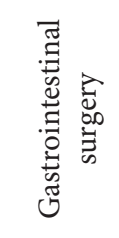 & 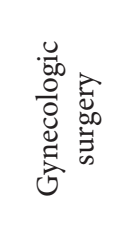 & 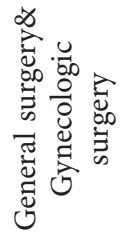 & 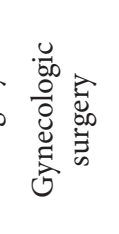 & 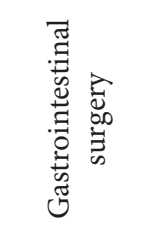 & 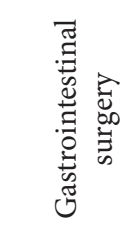 & 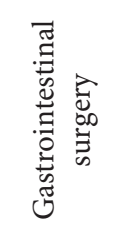 & 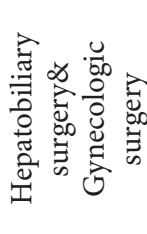 \\
\hline 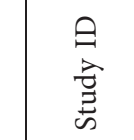 & 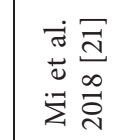 & 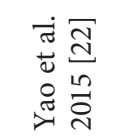 & 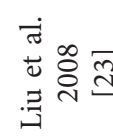 & 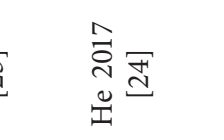 & 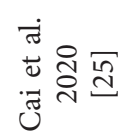 & 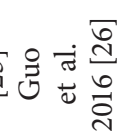 & ż $\frac{\bar{\pi}}{\mathrm{N}}$ & 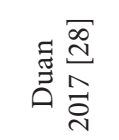 & $\begin{array}{l}\text { ड્ते } \\
\text { Jु }\end{array}$ & 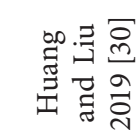 & 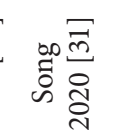 & 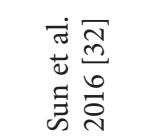 \\
\hline$\dot{z}$ & - & $N$ & $m$ & $r$ & in & 6 & $\Lambda$ & $\infty$ & $a$ & $\stackrel{ }{-1}$ & $\exists$ & $\simeq$ \\
\hline
\end{tabular}




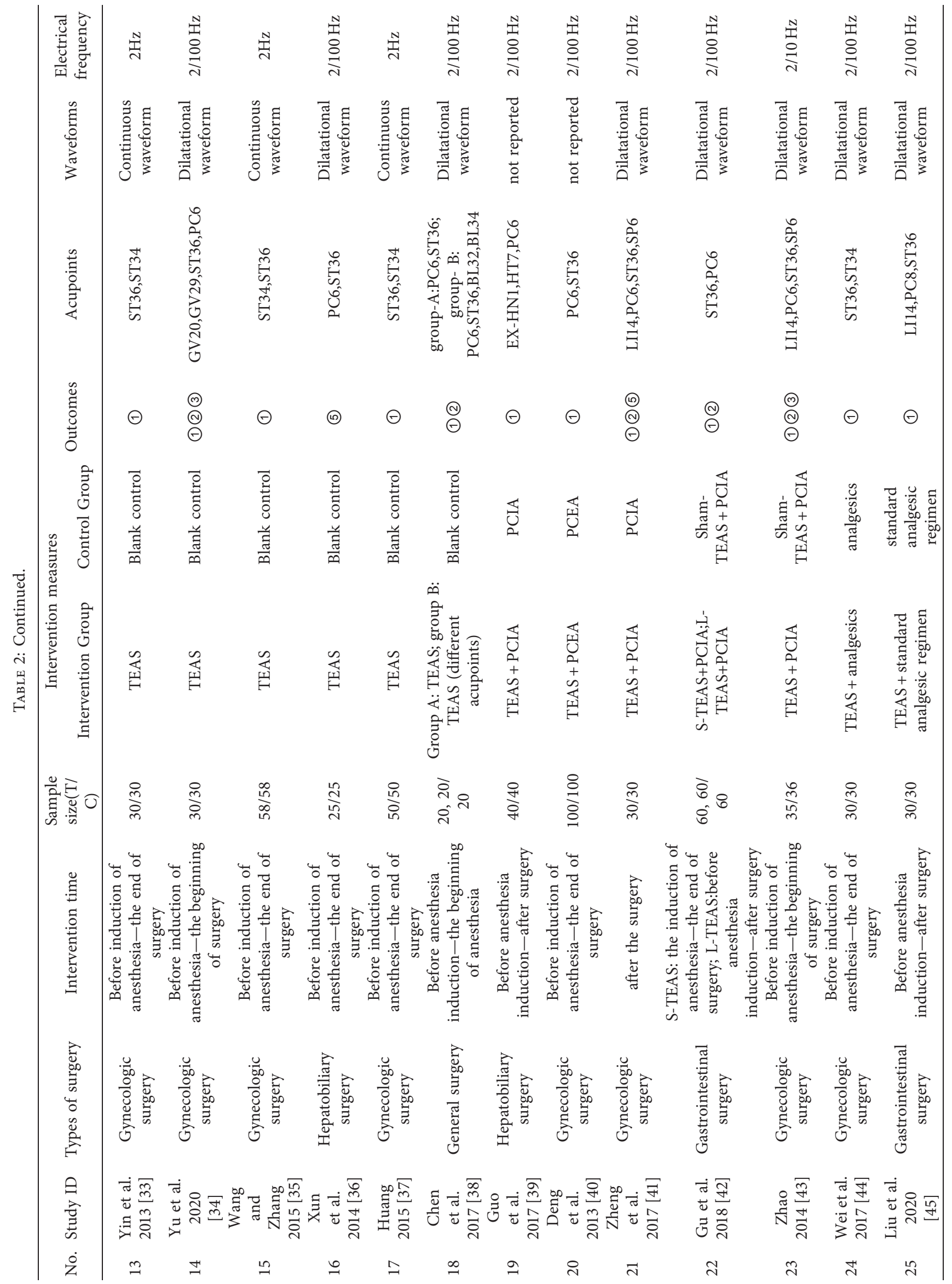




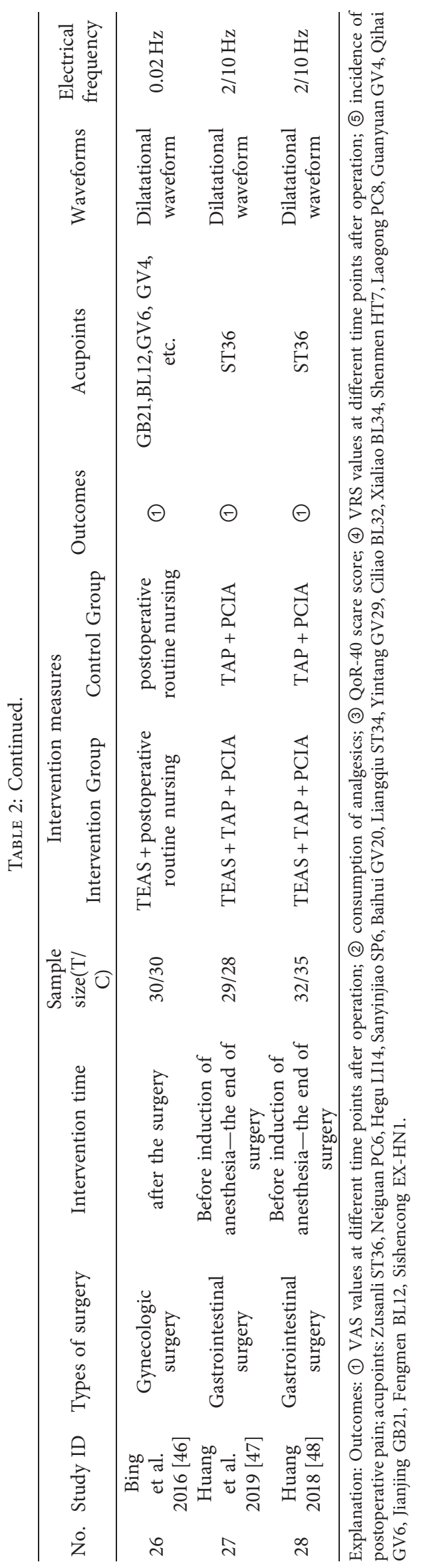




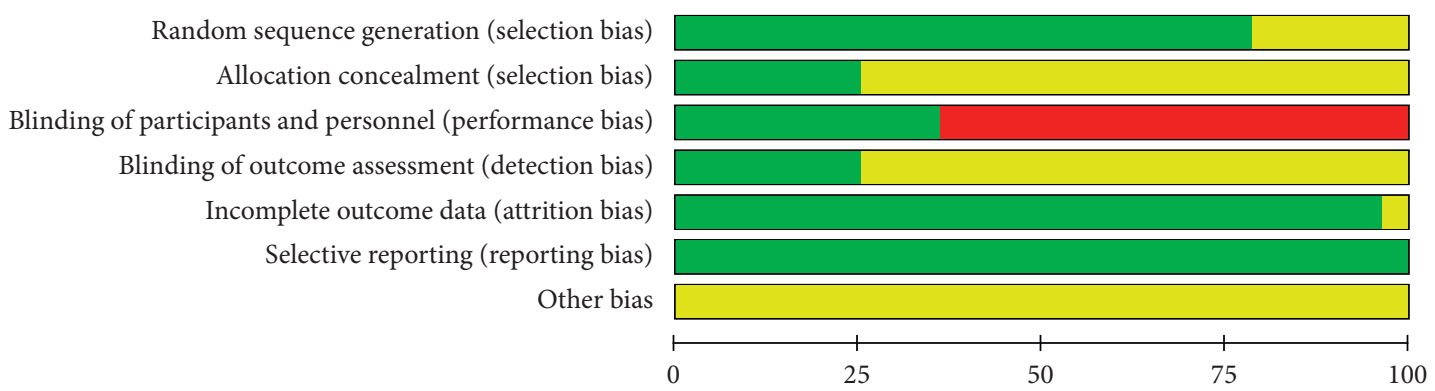

$(\%)$

Low risk of bias

Unclear risk of bias

High risk of bias

(a)

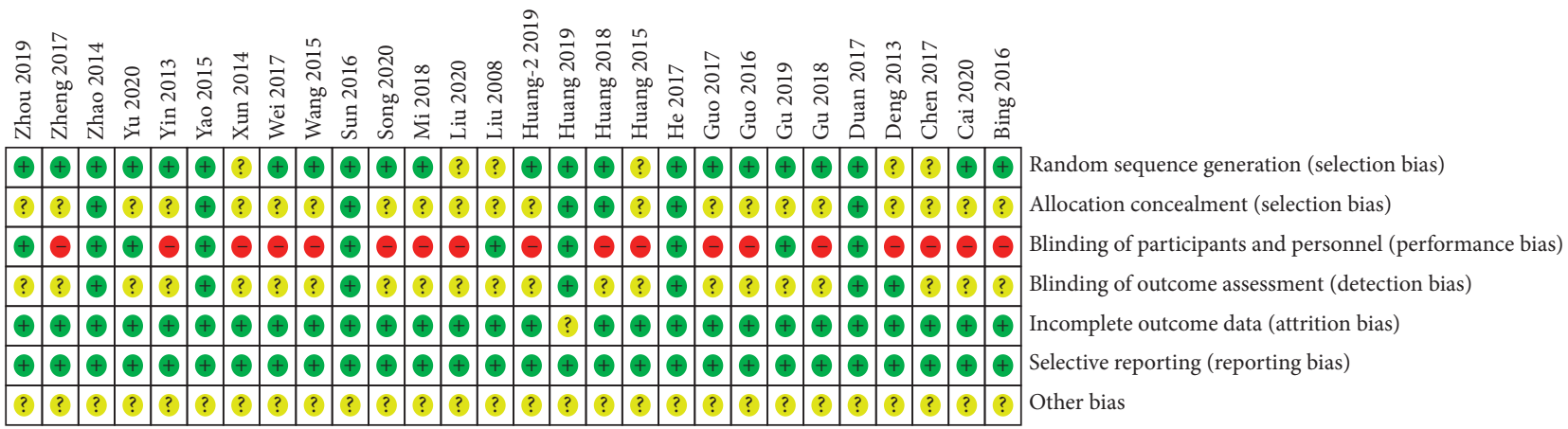

(b)

Figure 2: Risk of bias from included studies. (a) Risk of bias summary. Review authors' judgments about each risk of bias item for each included study. (b) Risk of bias graph. Review authors' judgments about each risk of bias item presented as percentages across all included studies.

\begin{tabular}{|c|c|c|}
\hline Study & & $\%$ \\
\hline ID & WMD (95\% CI) & Weight \\
\hline Huang 2018 & $-0.30(-0.63,0.03)$ & 11.72 \\
\hline Zheng 2017 & $-0.85(-1.40,-0.30)$ & 4.18 \\
\hline Gu 2019 & $-0.65(-0.84,-0.46)$ & 36.11 \\
\hline Gu 2018 & $-0.46(-0.65,-0.27)$ & 34.73 \\
\hline Cai 2020 & $-0.60(-0.91,-0.29)$ & 13.27 \\
\hline Overall $(\mathrm{I}$-squared $=26.1 \%, \mathrm{p}=0.248)$ & $-0.54(-0.66,-0.43)$ & 100.00 \\
\hline-1.4 & 1.4 & \\
\hline
\end{tabular}

FIGURE 3: Forest plot of VAS for postoperative pain intensity at $4 \mathrm{~h}$ in TEAS compared with control. 


\begin{tabular}{|c|c|c|c|}
\hline \multicolumn{4}{|l|}{ Study } \\
\hline ID & & WMD (95\% CI) & Weight $(\%)$ \\
\hline Huang 2019 & & $-0.34(-0.62,-0.06)$ & 17.26 \\
\hline Deng 2013 & $\longrightarrow$ & $0.30(0.10,0.50)$ & 17.66 \\
\hline Huang 2015 & & $-1.10(-1.36,-0.84)$ & 17.35 \\
\hline Wei 2017 & & $-0.70(-1.11,-0.29)$ & 16.33 \\
\hline Wang 2015 & & $-1.18(-1.76,-0.60)$ & 14.86 \\
\hline Bing 2016 & - & $-0.10(-0.48,0.28)$ & 16.54 \\
\hline Overall (I-squared $=94.0 \%, p=0.000)$ & & $-0.50(-1.02,0.02)$ & 100.00 \\
\hline NOTE: Weights are from random effects analysis & & & \\
\hline-1.76 & 0 & 1.76 & \\
\hline
\end{tabular}

FIGURE 4: Forest plot of VAS for postoperative pain intensity at $6 \mathrm{~h}$ in TEAS compared with control.

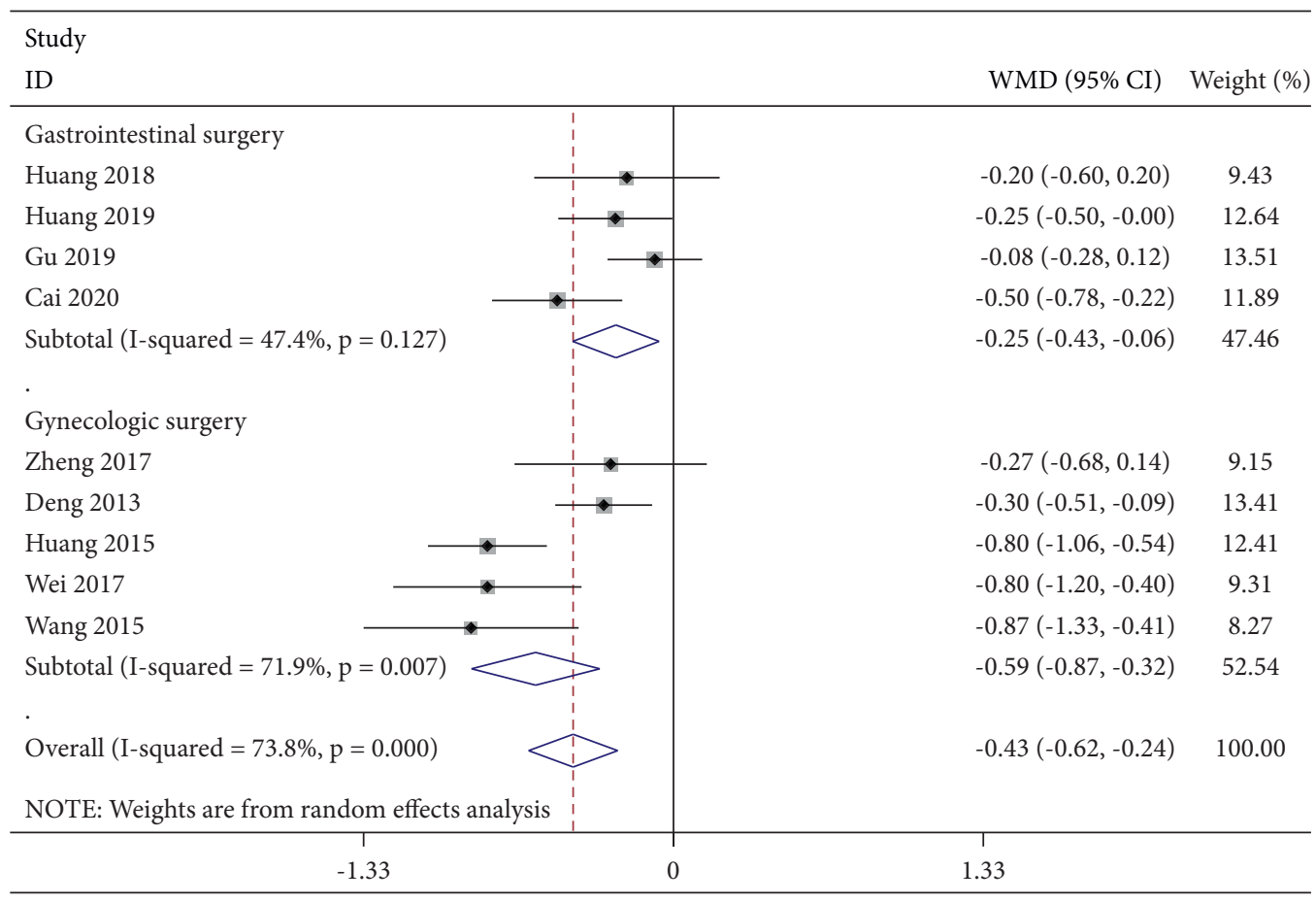

FIGURE 5: Forest plot of VAS for postoperative pain intensity at $12 \mathrm{~h}$ in TEAS compared with control.

3.50), $P<0.001$ ] within the TEAS group who underwent gynecological surgery.

3.5.2. Duration of Hospitalization. Five studies $[24,38,45,47,48]$ reported the duration of hospitalization. The fixed-effects model (Figure 9) was used with good homogeneity $\left(P=0.16, I^{2}=39.1 \%\right)$. Meta-analysis results indicated that the duration of hospitalization in the TEAS group was shorter than that in the control group $[\mathrm{SMD}=-0.51,95 \% \mathrm{CI}(-0.73,-0.29), P<0.001]$.
3.5.3. Adverse Events. Adverse events are the main evidence of safety evaluation. The adverse events in our study refer to a series of complications after the operation (e.g., nausea, vomiting, dizziness, pruritus, respiratory depression, etc.). In the 28 included studies, 6 [21, 28, 32, 36, 38, 44] of which reported postoperative adverse events, while the remaining studies did not mention anything about safety evaluation. Mi et al. [21] recorded the incidence of nausea and vomiting in the treatment and control groups at different time points after surgery. And the comparison between groups at $4 \mathrm{~h}$ and $8 \mathrm{~h}$ after surgery showed statistical advantage $(P<0.05)$, but 


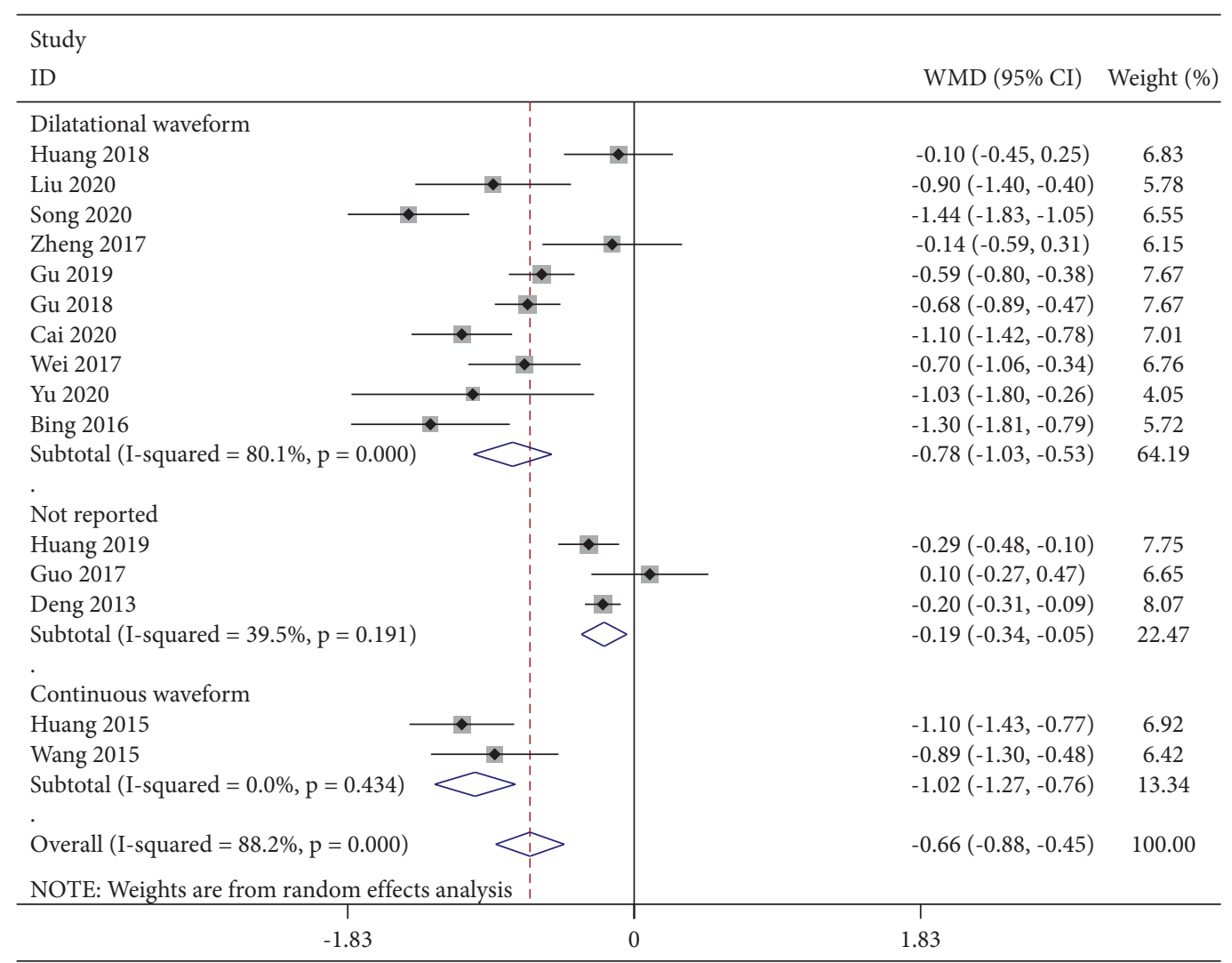

FIgURE 6: Forest plot of VAS for postoperative pain intensity at $24 \mathrm{~h}$ in TEAS compared with control.

Study
ID
Huang 2019
Zheng 2017
Gu 2019
Overall (I-squared $=0.0 \%, \mathrm{p}=0.943)$

FIGURE 7: Forest plot of postoperative consumption of analgesics at $12 \mathrm{~h}$ in TEAS compared with control.

there was no significant difference at $24 \mathrm{~h}$ and $48 \mathrm{~h}$ after surgery. The results of Chen et al. [38] showed, that compared with the control group, there was no case of vomiting and shivering in the treatment group, and there were only two cases of nausea. The results of 4 studies [28, 32, 36, 44] showed that no adverse events were observed throughout the procession of TEAS.
3.6. Sensitivity Analyses and Publication Bias. To assess the stability of the study results, our study performed a sensitivity analysis on the meta-analysis one by one. It showed that the results of each meta-analysis were stable. A funnel plot was used to assess publication bias based on the VAS score at 24 hours after the surgery $n \geq 10$ RCTs) (Figure 10). It showed that most of the points were asymmetrically 


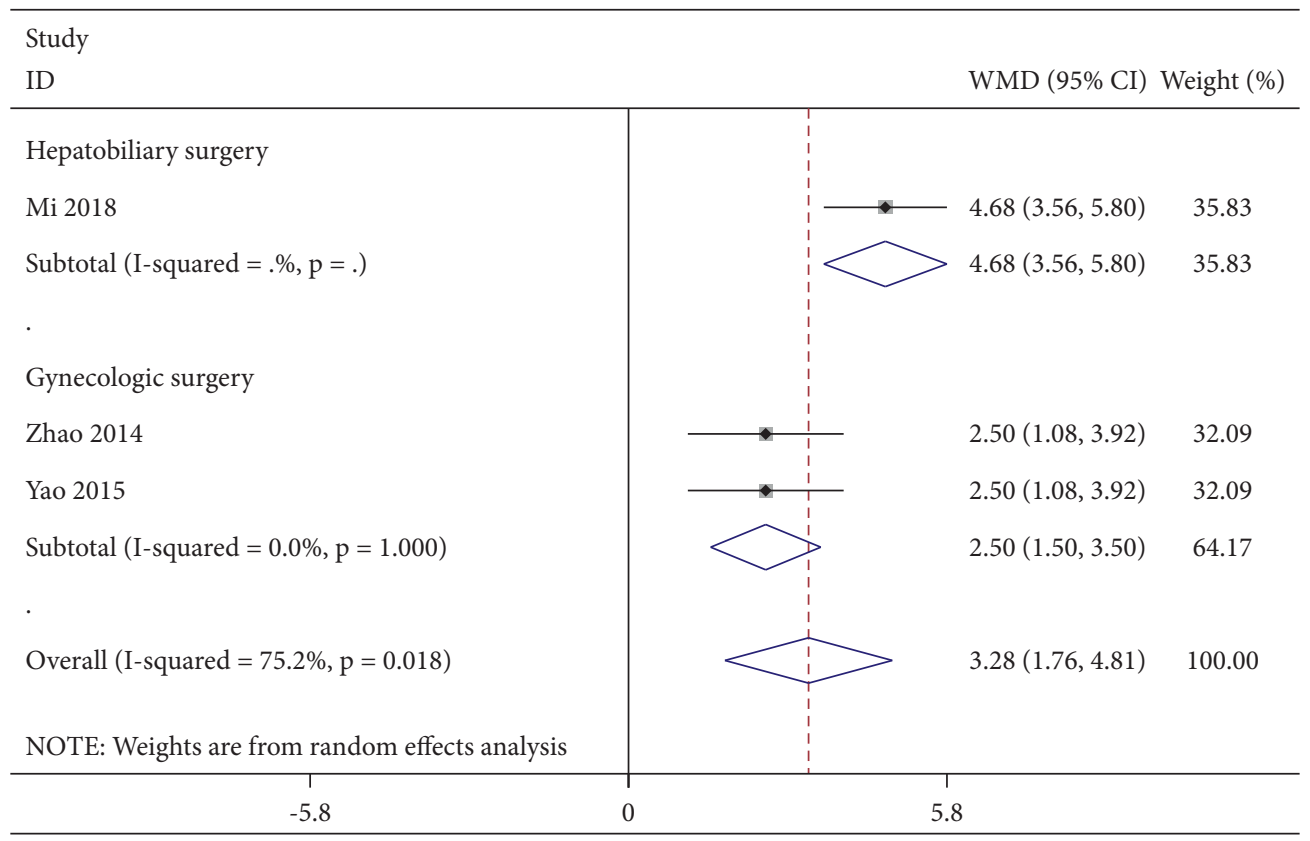

FIGURE 8: Forest plot of postoperative QoL-40 at $24 \mathrm{~h}$ in TEAS compared with control.

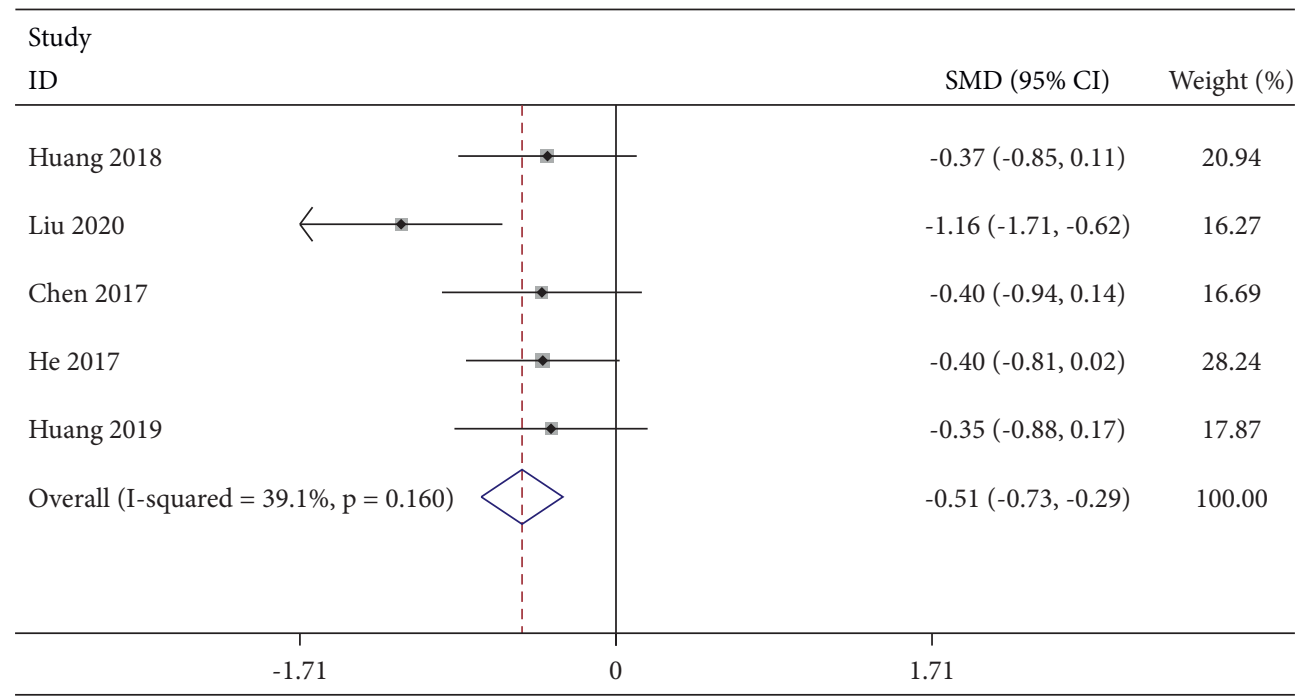

FIgURE 9: Forest plot of duration of hospitalization in TEAS compared with control.

distributed around, indicating that there maybe have been some publication bias. Consistently, Egger's test (Figure 11) suggested there is no publication bias in the RCTs $(P=0.261>0.05)$.

\section{Discussion}

Enhanced recovery after surgery (ERAS) advocates optimizing multiple approaches to perioperative pain management [49]. Postoperative analgesia is an important part of ERAS [50]. Nonincision pain is the most common complaint after laparoscopy, with an incidence of about 70\% 80\% [51]. The continuous administration of analgesics will reduce the pharmacological effectiveness [52], and a series of side effects will be produced by opioids. Therefore, there is an urgent need for optimized strategies to relieve postoperative pain after laparoscopy and improve the postoperative quality of life of patients.

To systematically assess the efficacy and safety of TEAS for postoperative pain after laparoscopy, our study conducted a systematic review and meta-analysis of 28 RCTs involving 2787 patients. Our meta-analysis suggests that TEAS can effectively relieve pain in the short term after laparoscopy, reduce the postoperative consumption of rescue analgesics, improve the quality of life of patients, and shorten the length of hospitalization. Therefore, TEAS can be recommended as a complementary and alternative therapy for the treatment of postoperative pain after 


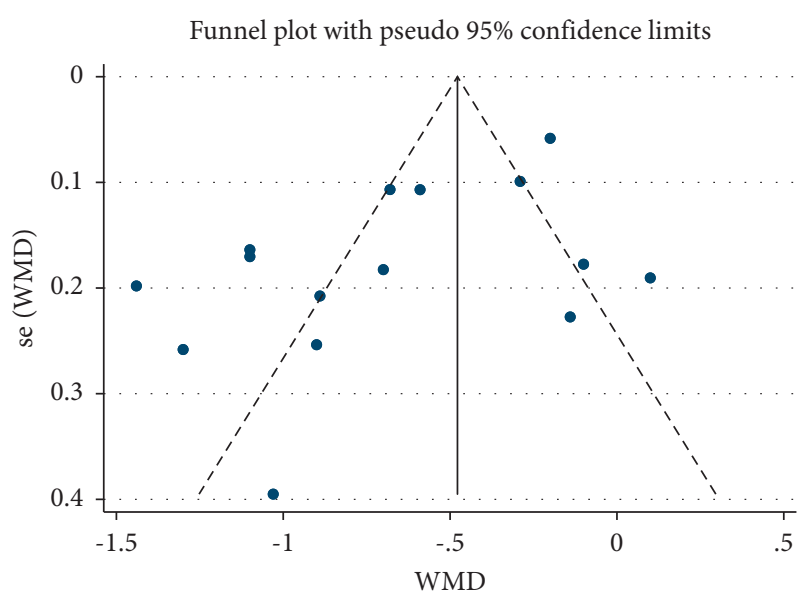

Figure 10: Funnel plot of publication bias.

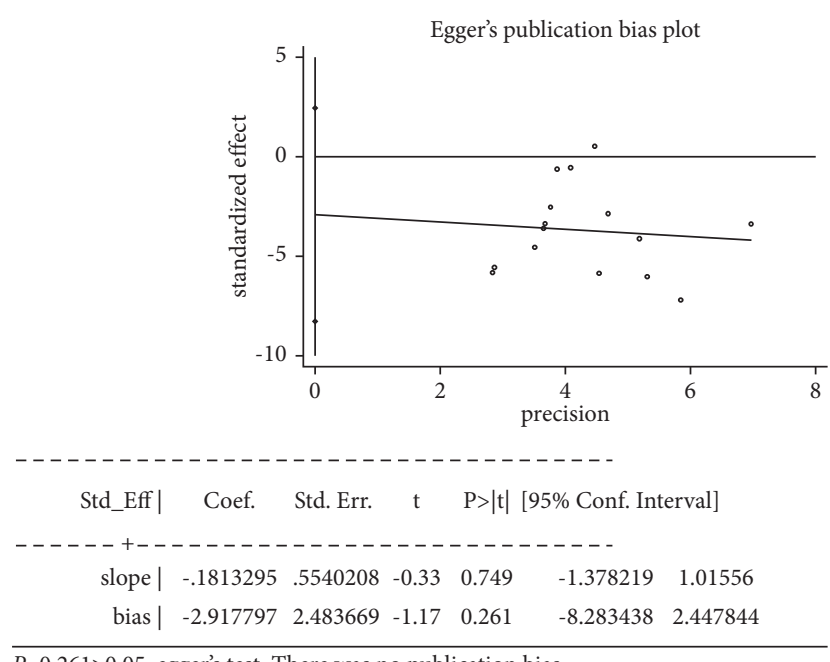

$P=0.261>0.05$, egger's test: There was no publication bias.

FIGURE 11: Egger's test of publication bias.

laparoscopy. Besides, no serious adverse events related to TEAS and the occurrence of treatment-related safety problems were reported in the included studies. Therefore, we could think cautiously that TEAS is relatively safe and efficacy for clinical application.

A previous study suggested that epidural anesthesia can reduce postoperative pain scores in abdominal surgery [53], so it is often regarded as a confounding factor for electroacupuncture or electrical stimulation to exert postoperative analgesia [54]. Therefore, our study only chose the operation of laparoscopy under general anesthesia, to avoid the interference of more potential factors. Pain intensity was selected as the outcome for it plays an important role in pain assessment and management [55]. VAS is commonly used in pain evaluation. Previous studies [56] have suggested that the subjectivity and multidimensionality of pain experience produce individual differences in pain assessment. Some studies showed that analgesic requirements are controlled by healthcare providers and are directly affected by the types of surgery and the economic status of patients. Analgesic consumption is not a particularly reliable indicator of the effectiveness of acupoint stimulation [57]. Therefore, more objective indicators, such as the metabolism of pain substances in the blood, will be needed for postoperative pain assessment in the future.

Besides, we did not categorize different types of pain, which may affect the results. Therefore, a more rigorous experimental design is needed to confirm the results of our study. We found that TEAS was effective in alleviating pain intensity at $4 \mathrm{~h}, 12 \mathrm{~h}$, and $24 \mathrm{~h}$ after laparoscopy, although this may depend on the type of surgery and waveform of the electrical stimulation device. TEAS can also effectively reduce the consumption of analgesics $12 \mathrm{~h}$ after surgery, but the sample size included in this study was small, and the pharmacological mechanism of different analgesics was not fully considered. This indicates that TEAS can effectively relieve postoperative pain in the short term. However, in the process of future studies, more objective large-scale RCTs are still needed to provide more evidence support, and more attention should be paid to the standardized research program of TEAS and the follow-up of long-term efficacy.

At present, the analgesic mechanism of TEAS is not clear. Some studies [58-60] believe that the treatment process can cause the release of neurotransmitters in some regions of the spinal cord and brain, which increases the endogenous analgesic substances such as opioid peptides, strong kephalins, enkephalins, and acetylcholine, thus raising the pain threshold. It has been suggested that electrical stimulation can block pain signals by activating the descending inhibitory system in the brain stem [61]. It has been suggested that electrical stimulation activates different functional areas of the cerebral cortex, thereby reducing the body's sensitivity to painful stimuli by affecting the corresponding innervation of the skin [62].

For patients who have experienced laparoscopy, the body function is in a relatively weak state, resulting in patients with insufficient strength, blood stasis, and reduced meridians, resulting in postoperative pain symptoms. According to the traditional meridian theory of traditional Chinese medicine, the regulating effect of acupuncture and moxibustion is reflected not only in regulating the movement of Qi and blood, making the meridians and collaterals smooth, but also in regulating the ups and downs of Qi and blood, so that the organs and collaterals are supported by Qi and blood, and correcting the partial decline of the body. Acupoints are special parts of the viscera by draining Qi into the body surface. Electrical stimulation on the acupoints can play the role of regulating Qi and regulating blood, draining meridians, and dredging collaterals. Hegu (LI14), Neiguan (PC6), and Zusanli (ST36) are important acupoints for analgesia in meridian theory. Both ancient literature and modern studies show that Hegu (LI14) is good at relieving pain [63]. The experimental study [61] showed that the plasma concentration of 5-hydroxytryptamine (5-HT) decreased after electroacupuncture of Hegu (LI14) in rabbits, to achieve the purpose of analgesia. It can play an analgesic role in many ways, such as increasing the body pain threshold, alleviating microvascular spasms, and influencing the frontal cortex to participate in the discharge of the pain unit in the ventrolateral thalamic nucleus [64]. Besides, it has 
been reported to be effective in treating acute pain in various parts of the body [65]. Neiguan (PC6) has the effect of acute pain in various parts of the body [66]. Zusanli (ST36) has the functions of health care, immune regulation, strengthening and dispelling pathogenic factors, and regulating the spleen and stomach. Electroacupuncture stimulation of bilateral Zusanli (ST36) in rats can stimulate the parasympathetic nerve and cholinergic pathway, which can effectively promote the recovery of gastrointestinal function in rats $[67,68]$. These acupoints are convenient and accurate, are easy to implement, and have good treatment compliance.

The QoR-40 scale is designed to evaluate the quality of postoperative recovery. Its effectiveness has been verified clinically, and it is widely used to evaluate the quality of postoperative recovery [69]. The duration of hospitalization is also an important indicator of postoperative recovery. Our results are similar to those of the previous studies [54], which reported that TEA was effective in reducing the duration of hospitalization $\left(\mathrm{MD}=1.30,95 \% \mathrm{CI}(-2.10,-0.51), I^{2}=32 \%\right.$, REM). Although this study has found that TEAS can improve postoperative quality of life and shorten the duration of hospitalization, more large RCTs with objective results are needed to confirm these findings.

For the aspects of the waveform and electrical stimulation frequency, the included studies mostly adopted dilatational waveform with $2 / 100 \mathrm{~Hz}$. In terms of analgesia, a previous study [70] has suggested that the analgesic effect of alternating stimulation of density wave is better than that of simultaneous stimulation of different frequencies. TEAS used in $2 / 100 \mathrm{~Hz}$ induced the release of all opioid peptides and peptides with synergistic effects [71]. It can be seen that these research results are consistent with our data results. For the intervention time, most of the included studies were conducted from the time before anesthesia induction to after surgery. One study [72] has found that preoperative electroacupuncture therapy can relieve the tension and fear of patients and, to a certain extent, reduce the number of postoperative opioids for patients with mental stress. It has been shown that preoperative acupoint therapy interventions can reduce the need for opioids and opioid-related side effects after surgery $[73,74]$. However, one study [75] found that intraoperative treatment did not improve postoperative pain and did not reduce the amount of opioids needed. Besides, the brain will produce different responses with the different timing of acupuncture treatment, thus affecting the therapeutic effect [76]. Therefore, it is suggested that the study on the timing of TEAS intervention should be strengthened and the treatment plan should be improved.

\section{Limitations}

It must be acknowledged that our meta-analysis has several limitations. (1) The intervention time, waveform, and frequency of electrical stimulation, acupoints, and other aspects of TEAS treatment are not uniform due to the different clinical experiences. At the same time, the treatment regimens in the control group were not uniform, which may affect the accuracy of the effectiveness assessment. (2) Our review may be affected by a high degree of heterogeneity, and it is difficult to find out the main reason that affects the efficacy of TEAS. (3) We did not distinguish between different types of pain and did not summarize the optimal TEAS treatment for postoperative laparoscopic pain. (4) Most RCTs were conducted in China, which may lead to publication bias and affect the validity and reliability of the system evaluation. (5) The methodological deficiencies and small sample size of the included trials may overestimate the efficacy to some extent. (6) No long-term follow-up information has been reported, so long-term efficacy remains to be confirmed.

\section{Conclusions}

In this systematic review and meta-analysis, TEAS can be cautiously considered as a treatment for postoperative pain after laparoscopy, which is effective in alleviating pain in the short term, reducing the use of remedial analgesics, and improving postoperative quality of life and length of hospital stay. TEAS is a noninvasive and easy-to-operate treatment, which can be operated after simple training, low application price, and good treatment compliance. It is worthy of clinical promotion and application. Although this study has limitations, it provides a direction and basis for future research. Due to the methodological deficiencies of the included RCTs, larger-size, more rigorous, and higher-quality RCTs are needed in the future to further explore the efficacy of TEAS in the treatment of postoperative pain after laparoscopy. Therefore, it is necessary to further optimize the treatment plan in terms of intervention time, electrical stimulation frequency and waveform, and acupoint selection. The longterm therapeutic effect of TEAS needs further studies determined by the observation of prolonged follow-up time. Besides, more attention should be paid to the economic impact of TEAS in the health care system.

\section{Data Availability}

(1) This systematic review and meta-analysis was performed in line with the Preferred Reporting Items for Systematic Reviews and Meta-Analyses (PRISMA) schema and was registered at INPLASY under registration number INPLASY202150101; the hyperlink is https://inplasy.com/ inplasy-2021-5-0101/. (2) The corresponding protocol has been published in Medicine; the doi is 10.1097/ MD.0000000000026348.

\section{Disclosure}

Dan Meng and Yifei Mao are the co-first authors.

\section{Conflicts of Interest}

The authors declare that there are no conflicts of interest concerning this work.

\section{Authors' Contributions}

D. Meng and Y. F. Mao are responsible for the meta-analysis study selecting, data extracting, manuscript writing, and 
manuscript editing. D. Meng is responsible for the metaanalysis study selecting and manuscript writing. All authors read and approved the final manuscript. D. Meng and Y.F. Mao contributed equally to this work.

\section{Acknowledgments}

This study was supported by the Fourth Batch of National Excellent Clinical Talents of Traditional Chinese Medicine Research and Training Program (National Education of Traditional Chinese Medicine [2017] No. 24) and Key Laboratory of Classical Theory of Traditional Chinese Medicine, Ministry of Education, Shandong University of Traditional Chinese Medicine, Jinan, 250355, China.

\section{Supplementary Materials}

In the supplementary files, there are seven sensitivity analyses, which are sensitivity analysis of VAS for postoperative $4 \mathrm{~h}, 6 \mathrm{~h}, 12 \mathrm{~h}$, and $24 \mathrm{~h}$, sensitivity analysis of QoR-40, sensitivity analysis of postoperative consumption of analgesics, and sensitivity analysis of duration of hospitalization. Sensitivity analysis can also be understood as robustness analysis, which is an important method mainly used to evaluate the robustness and reliability of the combined results of the meta-analysis. It is a common sensitivity analysis method to remove each included study one by one and then merge the effect quantity, changing the inclusion and exclusion criteria or removing a certain type of literature and then merging the effect quantity. Through the sensitivity analysis of this study, we can confirm that all results are stable and reliable. (Supplementary Materials)

\section{References}

[1] X. L. Yu, T. T. Yu, J. L. Lai, C. W. S, and Y. Xiao, "New progress in nursing of postoperative complications of gynecological laparoscopic patients," Nursing Practice and Research, vol. 12, no. 3, pp. 24-25, 2015.

[2] L. Z. Chen, "The shadow response of functional rehabilitation exercise exercise to the syndromes of pain after abdominal endoscopic surgery in gynecology," Medical forum, vol. 17, no. 29 , pp. 165-169, 2010.

[3] S. M. Shen, "Research progress in the prevention and treatment of neck and shoulder pain after gynecological laparoscopy," Clinical Progress, vol. 10, pp. 24-25, 2012.

[4] S.-H. Chang, H.-W. Lee, H.-K. Kim, S.-H. Kim, and D.-K. Kim, "An evaluation of perioperative pregabalin for prevention and attenuation of postoperative shoulder pain after laparoscopic cholecystectomy," Anesthesia \& Analgesia, vol. 109, no. 4, pp. 1284-1286, 2009.

[5] K. Slim, J. Bousquet, F. Kwiatkowski, G. Lescure, D. Pezet, and J. Chipponi, "Effect of $\mathrm{CO}_{2}$ gas warming on pain after laparoscopic surgery," Surgical Endoscopy, vol. 13, no. 11, pp. 1110-1114, 1999.

[6] V. L. Wills, D. R. Hunt, and A. Armstrong, "A randomized controlled trial assessing the effect of heated carbon dioxide for insufflation on pain and recovery after laparoscopic fundoplication," Surgical Endoscopy, vol. 15, no. 2, pp. 166-170, 2001.

[7] X. J. Pan, Y. P. Wang, and X. L. Wang, "Effect of knee and chest decubitus position and oxygen therapy on shoulder pain after gynecological laparoscopy," Chinese Journal of Nursing, vol. 45, no. 3, pp. 240-242, 2010.

[8] S. Dalal and E. Bruera, "Pain management for patients with advanced cancer in the opioid epidemic era," American Society of Clinical Oncology Educational Book, vol. 39, no. 39, pp. 24-35, 2019.

[9] Y. Sun, T. J. Gan, J. W. Dubose, and A. S. Habib, "Acupuncture and related techniques for postoperative pain: a systematic review of randomized controlled trials," British Journal of Anaesthesia, vol. 101, no. 2, pp. 151-160, 2008.

[10] M. S. Wu, K. H. Chen, I. F. Chen et al., "The efficacy of acupuncture in post-operative pain management: a systematic review and meta-analysis," PLoS One, vol. 11, no. 3, Article ID e0150367, 2016.

[11] S. Lee, J. Park, J. Kim et al., "Acupuncture for postoperative pain in laparoscopy: a systematic review protocol," BMJ Open, vol. 4, Article ID e006750, 2014.

[12] F. Lan, Y. H. Ma, J. X. Xue, T. L. Wang, and D. Q. Ma, "Transcutaneous electrical nerve stimulation on acupoints reduces fentany1 requirement for postoperative pain relief after total hip arthroplasty in elderly patients," Minerva Anestesiologica, vol. 78, no. 8, pp. 887-895, 2012.

[13] M. B. Silva, P. R. de Melo, N. M. L. de Oliveira, E. Crema, and L. F. R. M. Fernandes, "Analgesic effect of transcutaneous electrical nerve stimulation after laparoscopic cholecystectomy," American Journal of Physical Medicine \& Rehabilitation, vol. 91, no. 8, pp. 652-657, 2012.

[14] J. M. DeSantana, V. J. Santana-Filho, D. R. Guerra, K. A. Sluka, R. Q. Gurgel, and W. M. da Silva, "Hypoalgesic effect of the transcutaneous electrical nerve stimulation following inguinal herniorrhaphy: a randomized, controlled trial," The Journal of Pain, vol. 9, no. 7, pp. 623-629, 2008.

[15] M. A. R. B. Shovo, M. R. Tona, J. Mouah et al., "Computational and pharmacological studies on the antioxidant, thrombolytic, anti-inflammatory, and analgesic activity of molineria capitulata," Current Issues in Molecular Biology, vol. 43, no. 2, pp. 434-456, 2021.

[16] M. A. Freitas, A. Vasconcelos, E. C. D. Gonçalves et al., "Involvement of opioid system and TRPM8/TRPA1 channels in the antinociceptive effect of Spirulina platensis," Biomolecules, vol. 11, no. 4, p. 592, 2021.

[17] A. J. Chakraborty, S. Mitra, T. E. Tallei et al., "Bromelain a potential bioactive compound: a comprehensive overview from a pharmacological perspective," Life, vol. 11, no. 4, p. 317, 2021.

[18] J. Fernández, B. Silván, R. Entrialgo-Cadierno et al., “Antiproliferative and palliative activity of flavonoids in colorectal cancer," Biomedicine \& Pharmacotherapy = Biomedecine \& Pharmacotherapie, vol. 143, Article ID 112241, 2021.

[19] D. Meng, Y. Mao, Q. Song et al., "The efficacy and safety of transcutaneous electrical acupoint stimulation (TEAS) for postoperative pain in laparoscopy: a protocol for systematic review and meta-analysis of randomized controlled trials," Medicine, vol. 100, no. 25, Article ID e26348, 2021.

[20] J. P. T. Higgins, J. Thomas, J. Chandler et al., Cochrane Handbook for Systematic Reviews of Interventions Version 6.0, Cochrane, 2019, http://www.training.cochrane.org/handbook.

[21] Z. H. Mi, J. Gao, X. P. Chen, Y. Ge, and K. Lu, "Effect of transcutaneous acupoint electrical stimulation on the quality of early postoperative recovery in patients undergoing laparoscopic cholecystectomy," Chinese Acupuncture and Moxibustion, vol. 38, no. 3, pp. 256-260, 2018.

[22] Y. Yao, Q. Zhao, C. Gong et al., "Transcutaneous electrical acupoint stimulation improves the postoperative quality of 
recovery and analgesia after gynecological laparoscopy: a randomized controlled trial," Evidence-Based Complementary and Alternative Medicine, vol. 2015, Article ID 324360, 6 pages, 2015.

[23] Y.-y. Liu, S.-e. Duan, M.-x. Cai, P. Zou, Y. Lai, and Y.-1. Li, "Evaluation of transcutaneous electroacupoint stimulation with the train-of-four mode for preventing nausea and vomiting after laparoscopic cholecystectomy," Chinese Journal of Integrative Medicine, vol. 14, no. 2, pp. 94-97, 2008.

[24] S. He, Effects of Different Stimulation Duration on the Anesthetic Effect of Needle and Drug Balance: A Prospective, Randomized, Double-Blind Controlled Study, The Fourth Military Medical University, Xian, China, 2017.

[25] Q. X. Cai, M. Zhao, Y. H. Li, Y. K. Huang, and F. Li, “Application of transcutaneous acupoint electrical stimulation assisted anesthesia in accelerated rehabilitation surgery for endoscopic radical resection of colorectal cancer," Journal of New Chinese Medicine, vol. 52, no. 19, pp. 122-125, 2020.

[26] Y. Q. Guo, W. Wu, Z. L. Yang, Y. Q. Zhang, Y. C. Tai, and R. Z. Zheng, "Effect of transcutaneous acupoint electrical stimulation combined with general anesthesia on complications after laparoscopic ovarian cyst resection," Journal of Liaoning University of Traditional Chinese Medicine, vol. 18, no. 8, pp. 100-102, 2016.

[27] M. J. Zhou, Effect of Transcutaneous Acupoint Electrical Stimulation on Analgesic Effect and Stress Response in Patients Undergoing Laparoscopy, Beijing University of Chinese Medicine, Beijing, China, 2019.

[28] H. F. Duan, Effects of Single-point and Double-point TEAS on PONV in Gynecological Endoscopic Surgery: A Prospective, Randomized, Double-Blind, Controlled Study, The Fourth Military Medical University, Xian, China, 2017.

[29] S. H. Gu, Comparison of Analgesic Effect and Gastrointestinal Function Recovery after Laparoscopic Radical Gastrectomy by Transcutaneous Acupoint Stimulation at a Different Time during the Perioperative Period, North China University of Science and Technology, Tangshan, China, 2019.

[30] Y. K. Huang and H. H. Liu, "Effects of acupoint electrical stimulation assisted anesthesia on gastrointestinal hormone and immune function in patients undergoing endoscopic rectal cancer surgery," Journal of Xinjiang Medical University, vol. 42, no. 12, pp. 1630-1634, 2019.

[31] K. Song, Effect of Transcutaneous Acupoint Electrical Stimulation on Gastrointestinal Function in Patients Undergoing Laparoscopic Gastrectomy, Wannan Medical College, Wuhu, China, 2020.

[32] K. Sun, T. Xing, F. Zhang et al., "Perioperative transcutaneous electrical acupoint stimulation for postoperative pain relief following laparoscopic surgery," The Clinical Journal of Pain, vol. 33, no. 4, pp. 340-347, 2017.

[33] X. Q. Yin, Y. C. Zhou, H. Zhou, H. Yang, Y. Q. Wang, and H. Zhang, "Transcutaneous acupoint electrical stimulation assisted general anesthesia can reduce the postoperative reaction of gynecological laparoscopy," Journal of Acupuncture Research, vol. 38, no. 6, pp. 431-434, 2013.

[34] X. Yu, F. Zhang, and B. Chen, "The effect of TEAS on the quality of early recovery in patients undergoing gynecological laparoscopy: a prospective, randomized, placebo-controlled trial," Trials, vol. 21, no. 8, p. 43, 2020.

[35] Y. A. Wang and L. M. Zhang, "Effect of transcutaneous acupoint electrical stimulation assisted general anesthesia on reducing postoperative reaction of gynecological laparoscopy," Journal of Modern Integrated Traditional and Western Medicine, vol. 24, no. 17, pp. 1903-1905, 2015.
[36] S. N. Xun, Y. Q. Zhang, and H. G. Song, "Clinical study of transcutaneous acupoint electrical stimulation combined with general anesthesia in laparoscopic cholecystectomy," Journal of Xuzhou Medical College, vol. 34, no. 4, pp. 249-251, 2014.

[37] J. S. Huang, "Effect of transcutaneous acupoint electrical stimulation on the postoperative response of gynecological laparoscopy," Journal of New Chinese Medicine, vol. 47, no. 7, pp. 246-247, 2015.

[38] Y. Chen, C. Y. Liu, H. M. Xu, and W. Q. Tian, "Effect of TEAS at different acupoints before anesthesia on analgesia and rehabilitation in transumbilical single-port laparoscopic radical resection of prostate cancer," Journal of Traditional Chinese Medicine, vol. 49, no. 12, pp. 59-61, 2017.

[39] B. Guo, L. Xie, T. Deng, and L. Deng, "Effect of electrotherapy on early cognitive dysfunction in elderly patients undergoing general anesthesia," Journal of Emergency in Traditional Chinese Medicine, vol. 26, no. 6, pp. 1083-1086, 2017.

[40] L. Deng, X. C. Yang, T. Deng, W. Tang, and F. Chen, "Effect of transcutaneous acupoint electrical stimulation on nausea and vomiting after gynecological laparoscopy," Journal of Emergency in Traditional Chinese Medicine, vol. 22, no. 6, pp. 902-904, 2013.

[41] R. Z. Zheng and Y. Q. Zhang, "Effect of transcutaneous acupoint electrical stimulation on postoperative pain after a laparoscopic total hysterectomy," Journal of Guangxi Medical University, vol. 34, no. 11, pp. 1652-1654, 2017.

[42] S. H. Gu, J. H. Gan, and H. B. Lang, "Comparison of analgesic effects and gastrointestinal function recovery after laparoscopic radical gastrectomy by different transcutaneous acupuncture point stimulation methods," Journal of Fujian Medical University, vol. 52, no. 2, pp. 125-129, 2018.

[43] Q. Y. Zhao, Preoperative Transcutaneous Acupoint Electrical Stimulation Improves Postoperative Recovery Quality of Patients Undergoing Gynecological Laparoscopy, Fujian Medical University, Fujian, China, 2015.

[44] Y. R. Wei, Y. Q. Zhang, S. N. Xun, W. H. Lu, and X. Q. Zhang, "Effect analysis of dexmedetomidine combined with transcutaneous acupoint stimulation in analgesia in gynecological laparoscopy," Journal of modern traditional Chinese medicine, vol. 37, no. 1, pp. 32-34, 2017.

[45] Z. Y. Liu, Z. P. Song, and F. Tang, "Effect of transcutaneous acupoint electrical stimulation on immune function in patients undergoing laparoscopic radical gastrectomy," Journal of Jiangxi Medical, vol. 55, no. 5, pp. 508-509, 2020.

[46] B. X. Bing, Z. X. Wang, and Z. H. Liang, "Effect of transcutaneous acupoint electrical stimulation on relieving nonincision pain after gynecological laparoscopy," Journal of Clinical Rational Drug Use, vol. 9, no. 21, pp. 118-119, 2016.

[47] W. Huang, W. Long, J. Xiao, G. Zhao, and T. Yu, "Effect of electrically stimulating acupoint, Zusanli (ST 36), on patient's recovery after laparoscopic colorectal cancer resection: a randomized controlled trial," Journal of Traditional Chinese Medicine $=$ Chung $i$ Tsa Chih Ying Wen Pan, vol. 39, no. 3, pp. 433-439, 2019.

[48] W. Huang, Application of Transcutaneous Acupoint Electrical Stimulation in Accelerated Rehabilitation Surgery, Guangzhou University of Traditional Chinese Medicine, Guangzhou, China, 2018.

[49] M. Cannesson and Z. Kain, "Enhanced recovery after surgery versus perioperative surgical home," Anesthesia \& Analgesia, vol. 118, no. 5, pp. 901-902, 2014.

[50] P. F. White, H. Kehlet, J. M. Neal, T. Schricker, D. B. Carr, and F. Carli, "The role of the anesthesiologist in fast-track surgery: 
from multimodal analgesia to perioperative medical care," Anesthesia \& Analgesia, vol. 104, no. 6, pp. 1380-1396, 2007.

[51] F. F. Xu, L. B. Xiao, J. D. Zuo et al., "Shoulder pain after abdominal laparoscopic operation: a multicenter study," Chinese Medical Journal, vol. 126, no. 2, pp. 382-384, 2013.

[52] N. I. Cherny and R. K. Portenoy, "Cancer pain management. The current strategy," Cancer, vol. 72, no. 11, pp. 3393-3415, 1993.

[53] Z. Q. Meng, M. K. Garcia, J. S. Chiang et al., "Electro-acupuncture to prevent prolonged postoperative ileus: a randomized clinical trial," World Journal of Gastroenterology, vol. 16, no. 1, pp. 104-111, 2010.

[54] K.-B. Chen, Y. Huang, X.-L. Jin, and G.-F. Chen, "Electroacupuncture or transcutaneous electroacupuncture for postoperative ileus after abdominal surgery: a systematic review and meta-analysis," International Journal of Surgery, vol. 70, pp. 93-101, 2019.

[55] R. L. Fainsinger, A. Fairchild, C. Nekolaichuk, P. Lawlor, S. Lowe, and J. Hanson, "Is pain intensity a predictor of the complexity of cancer pain management?" Journal of Clinical Oncology, vol. 27, no. 4, pp. 585-590, 2009.

[56] Y. Yang, J. Wen, and J. Hong, "The effects of auricular therapy for cancer pain: a systematic review and meta-analysis," Evidence-Based Complementary and Alternative Medicine, vol. 2020, Article ID 1618767, 10 pages, 2020.

[57] X. L. Liu, J. Y. Tan, A. Molassiotis, L. K. P. Suen, and Y. Shi, "Acupuncture-point stimulation for postoperative pain control: a systematic review and meta-analysis of randomized controlled trials," Evidence-Based Complementary and Alternative Medicine, vol. 2015, Article ID 657809, 28 pages, 2015.

[58] H. Ruan, X. W. Li, and W. Q. Cai, "Effects of 5-Hydroxytryptamine and somatostatin on substance $\mathrm{P}$ and the electrical activity of spinal dorsal horn neurons induced by chronic pain," Journal of acupuncture research, vol. 21, no. 3, p. 27, 1996.

[59] F. R. Liang, Y. X. Liu, and J. Chen, "The relationship between electroacupuncture analgesia effect and the content of 5-HT, hypothalamus $\beta$-EP in the brain stem," Shanghai Journal of Acupuncture and Moxibustion, vol. 3, pp. 37-39, 2001.

[60] X. F. Tian and M. F. Ren, "The role of opioid peptides and related factors in spinal cord injury," Journal of Advances in Physiological Sciences, vol. 3, no. 3, p. 214, 1992.

[61] X. L. Chen and K. Y. Song, "The analgesic effect of electroacupuncture at Hegu point in rabbits with soft tissue injury at different hours and its effect on plasma 5-hydroxytryptamine," Journal of Chengdu University of Traditional Chinese Medicine, vol. 24, no. 3, p. 25, 2001.

[62] X. W. Han, Z. Z. Huang, and Y. X. Lu, "Cortex stimulation or frozen block body feeling I area to the operation of the monkey conditioned reflex and the influence of acupuncture analgesic action," Journal of physiology, vol. 34, no. 2, pp. 165-172, 1982.

[63] C. H. Li, Y. Z. Wang, and X. X. Guo, "Clinical observation on primary dysmenorrhea treated mainly by needling SiGuan point," Chinese Acupuncture and Moxibustion, vol. 28, no. 3, p. 187, 2008.

[64] X. T. Ma, W. Zhai, and Y. X. Liu, "Literature study on acupoint selection rule of acupuncture anesthesia in thyroid surgery," Liaoning Journal of Traditional Chinese Medicine, vol. 42, no. 12, pp. 2401-2403, 2015.

[65] H. Lin, C. L. Lu, J. M. Wu, and J. Li, "Literature study of NeiGuan acupoint in the treatment of pain," Journal of
Chengdu University of Traditional Chinese Medicine, vol. 27, no. 4, pp. 57-59, 2004.

[66] Y. Yang and M. Q. Zhou, "Clinical effect of NeiGuan acupoint," Clinical Journal of Traditional Chinese Medicine, vol. 24, no. 7, pp. 614-615, 2012.

[67] J. Song, J. Yin, H. S. Sallam, T. Bai, Y. Chen, and J. D. Chen, "Electroacupuncture improves burn-induced impairment in gastric motility mediated via the vagal mechanism in rats," Neuro-Gastroenterology and Motility: The Official Journal of the European Gastrointestinal Motility Society, vol. 25, no. 10, p. 807, Article ID e635, 2013.

[68] Y. S. Su, W. He, C. Wang et al., "Intensity-response effects of electroacupuncture on gastric motility and its underlying peripheral neural mechanism," Evidence-Based Complementary and Alternative Medicine, vol. 2013, Article ID 535742, 8 pages, 2013.

[69] B. F. Gornall, P. S. Myles, C. L. Smith et al., "Measurement of quality of recovery using the QoR-40: a quantitative systematic review," British Journal of Anaesthesia, vol. 111, no. 2, pp. 161-169, 2013.

[70] J.-S. Han, "Acupuncture: neuropeptide release produced by electrical stimulation of different frequencies," Trends in Neurosciences, vol. 26, no. 1, pp. 17-22, 2003.

[71] J. S. Han, "Acupuncture and endorphins," Neuroscience Letters, vol. 361, no. 1-3, pp. 258-261, 2004.

[72] G. V. Chernyak, D. I. Sessler, and D. C. Warltier, "Perioperative acupuncture and related techniques," Anesthesiology, vol. 102, no. 5, pp. 1031-1049, 2005.

[73] J.-G. Lin, M.-W. Lo, Y.-R. Wen, C.-L. Hsieh, S.-K. Tsai, and W.-Z. Sun, "The effect of high and low frequency electroacupuncture in pain after lower abdominal surgery," Pain, vol. 99, no. 3, pp. 509-514, 2002.

[74] C. K. Sim, P. C. Xu, H. L. Pua, G. J. Zhang, and T. L. Lee, "Effects of electroacupuncture on intraoperative and postoperative analgesic requirement," Acupuncture in Medicine, vol. 22, no. 2-3, pp. 56-65, 2002.

[75] M. El-Rakshy, S. C. Clark, J. Thompson, and M. Thant, "Effect of intraoperative electroacupuncture on postoperative pain, analgesic requirements, nausea and sedation: a randomised controlled trial," Acupuncture in Medicine, vol. 27, no. 1, pp. 9-12, 2009.

[76] W. Qin, L. Bai, J. Dai et al., “The temporal-spatial encoding of acupuncture effects in the brain," Molecular Pain, vol. 7, p. 19, 2011. 